\title{
Transcriptome analysis reveals potential function of long non-coding RNAs in 20- hydroxyecdysone regulated autophagy in Bombyx mori
}

Huili Qiao ${ }^{1}$, Jingya Wang ${ }^{1,2}$, Yuanzhuo Wang ${ }^{1}$, Juanjuan Yang ${ }^{1}$, Bofan Wei ${ }^{1}$, Miaomiao Li ${ }^{1,2}$, Bo Wang ${ }^{1}$, Xiaozhe Li ${ }^{1}$, Yang $\mathrm{CaO}^{3,4}$, Ling $\operatorname{Tian}^{4}$, Dandan $\mathrm{Li}^{1}$, Lunguang $\mathrm{YaO}^{1}$ and Yunchao Kan ${ }^{1 *}$

\begin{abstract}
Background: 20-hydroxyecdysone (20E) plays important roles in insect molting and metamorphosis. 20E-induced autophagy has been detected during the larval-pupal transition in different insects. In Bombyx mori, autophagy is induced by 20E in the larval fat body. Long non-coding RNAs (IncRNAs) function in various biological processes in many organisms, including insects. Many IncRNAs have been reported to be potential for autophagy occurrence in mammals, but it has not been investigated in insects.

Results: RNA libraries from the fat body of B. mori dissected at 2 and $6 \mathrm{~h}$ post-injection with 20E were constructed and sequenced, and comprehensive analysis of IncRNAs and mRNAs was performed. A total of 1035 IncRNAs were identified, including 905 lincRNAs and 130 antisense IncRNAs. Compared with mRNAs, IncRNAs had longer transcript length and fewer exons. 132 IncRNAs were found differentially expressed at $2 \mathrm{~h}$ post injection, compared with 64 IncRNAs at $6 \mathrm{~h}$ post injection. Thirty differentially expressed IncRNAs were common at 2 and $6 \mathrm{~h}$ postinjection, and were hypothesized to be associated with the 20E response. Target gene analysis predicted 6493 IncRNA-mRNA cis pairs and 42,797 IncRNA-mRNA trans pairs. The expression profiles of LNC_000560 were highly consistent with its potential target genes, Atg4B, and RNAi of LNC_000560 significantly decreased the expression of LNC_000560 and Atg4B. These results indicated that LNC_000560 was potentially involved in the 20E-induced autophagy of the fat body by regulating Atg4B.

Conclusions: This study provides the genome-wide identification and functional characterization of IncRNAs associated with 20E-induced autophagy in the fat body of B. mori. LNC_000560 and its potential target gene were identified to be related to 20-regulated autophagy in B. mori. These results will be helpful for further studying the regulatory mechanisms of IncRNAs in autophagy and other biological processes in this insect model.
\end{abstract}

Keywords: LncRNA, Transcriptome, 20-hydroxyecdysone, Autophagy-related gene, Silkworm

\footnotetext{
* Correspondence: yckan1974@nynu.edu.cn

${ }^{1}$ China-UK-NYNU-RRes Joint Laboratory of insect biology, Henan Key

Laboratory of Insect Biology in Funiu Mountain, Nanyang Normal University,

473061 Nanyang, Henan, China

Full list of author information is available at the end of the article
}

(c) The Author(s). 2021 Open Access This article is licensed under a Creative Commons Attribution 4.0 International License, which permits use, sharing, adaptation, distribution and reproduction in any medium or format, as long as you give appropriate credit to the original author(s) and the source, provide a link to the Creative Commons licence, and indicate if changes were made. The images or other third party material in this article are included in the article's Creative Commons licence, unless indicated otherwise in a credit line to the material. If material is not included in the article's Creative Commons licence and your intended use is not permitted by statutory regulation or exceeds the permitted use, you will need to obtain permission directly from the copyright holder. To view a copy of this licence, visit http://creativecommons.org/licenses/by/4.0/. The Creative Commons Public Domain Dedication waiver (http://creativecommons.org/publicdomain/zero/1.0/) applies to the data made available in this article, unless otherwise stated in a credit line to the data. 


\section{Background}

Macroautophagy (hereafter autophagy) is an essential, evolutionarily conserved cellular degradation and recycling process in all eukaryotes [1]. The role of autophagy is to maintain cellular homeostasis by degrading intracellular components. Autophagy is a process involving induction, cargo recognition and packaging, vesicle formation, and breakdown. A series of autophagy-related (Atg) genes are required for the initiation, nucleation, expansion, and completion of bodies known as autophagosomes, which eventually fuse with lysosomes [2]. Autophagy is essential to many physiological and developmental processes, and defects in autophagy are often associated with diseases and tumor progression $[3,4]$.

Autophagy is regulated by several ATG proteins, which are evolutionary conserved from yeast to mammals, ATG proteins are classified into six functional complexes including ATG1-kinase complex, phosphatidylinositol-3-kinase complex, ATG2-ATG18 complex, ATG9 membrane protein, ATG8 conjugation system and ATG12 conjugation system [5]. ATG4 is the only cysteine protease specific to ATG8, and essential for the conjugation and deconjugation of ATG8. Although ATG4 and ATG8 are evolutionarily conserved, higher eukaryotes have multiple homologs for both proteins. In contrast to the Atg4 and Atg8 in yeast, there are four ATG4 and six ATG8 homologs in mammals, the protease activity of the ATG4 homologs is markedly different, but ATG4B exhibits much higher activity than the other homologs [6, 7]. ATG4 homologs are important for autophagosome formation, autophagy is inhibited by suppressing ATG4 expression [8]. In B. mori, 15 Atgs have been identified in the genome $[9,10]$, and include two Atg4 homologs, Atg4B and Atg4-like, but their function are still not characterized.

In insects, autophagy is an important physiological process during metamorphosis. The molting and metamorphosis of insects are regulated primarily by 20hydroxyecdysone (20E) and juvenile hormone $(\mathrm{JH})[11,12]$. 20E-induced autophagy can be detected during the larvalpupal transition in different insects as well as in B. mori. In the fat body, the ecdysone receptor (EcR) is necessary for the induction of autophagy by $20 \mathrm{E}$ in Drosophila melanogaster and B. mori, while Atg genes are upregulated in B. mori during molting and pupation $[10,13,14]$.

Long non-coding RNAs (lncRNAs) are a large class of RNA transcripts that are longer than 200 nucleotides and lack protein-coding potential [15]. The majority of IncRNAs are transcribed by RNA polymerase II, and processed by 5'-capping, 3'-polyadenylation, and alternative splicing, similar to mRNAs [16]. In the last decades, advances in transcriptome sequencing have led to the identification of a large number of IncRNAs in various eukaryotic organisms using new technologies and bioinformatics methods [17-22], but there are still few studies into their functions. Recently, lncRNAs have attracted attention because of their critical roles in organismal growth, development, senescence, and death. There is evidence that lncRNAs participate in a range of biological processes, such as X-chromosome silencing [23], dosage compensation [24], chromosome modification [25], genomic imprinting [26], and control of gene expression [27].

Several studies have investigated the regulatory mechanisms of lncRNAs in autophagy from multiple aspects in mammals. For example, high glucose levels have been shown to reduce the expression of the IncRNA H19, which activates the transcription of DIRAS3 and induces autophagy by repressing the PI3K/AKT/mTOR pathway [28]. LncRNAs HOTAIRM promote the initiation of autophagy by increasing ULK expression [29]. The lncRNAs PCGEM1 affect the nucleation of autophagy by regulating the expression of Beclin1 [30]. The lncRNAs $A P F$ can promote the extension and fusion of autophagosomes [31]. The IncRNA MALAT1 can also regulate the expression of $L A M P 1$ by miR-23-3p during the formation of autolysosomes [32]. However, research into insect lncRNAs and their functions during metamorphosis and autophagy is still scarce.

The silkworm B. mori, an economically important insect which undergoes complete metamorphosis, is a good model to study the role of lncRNAs in autophagy. The fat body is an organ which is important for nutrient storage and energy metabolism in insects. It plays critical roles in the regulation of insect metamorphosis by coordinating different hormones and nutritional signals $[33,34]$. Previous studies have shown that the injection of $20 \mathrm{E}$ into actively feeding larvae up-regulates Atg genes and reduces TORC1 activity, inducing autophagy in the fat body [10]. Although some progress has recently been made on the investigation of silkworm lncRNAs [3541], their functions remain poorly understood, including whether and how they regulate autophagy and metamorphosis. In this study, the lncRNAs associated with the $20 \mathrm{E}$ response in the fat body of $B$. mori were identified and validated. The function of the selected lncRNA was further characterized by studying the specific expression patterns and its target gene. To the best of our knowledge, our study was the first to identify lncRNA that might be involved in the 20E-induced autophagy in silkworm. Our results lay a foundation for future studies in elucidating the regulatory role of lncRNAs in autophagy and other biological processes in B. mori.

\section{Results}

Genome-wide identification of IncRNAs using RNA-seq

We first estimated the 20E-induced autophagy in the fat body from the 2-day-old 5th instar larvae using LysoTracker Red staining. The staining was undetectable in 
the control samples. However, in the 20E-induced samples, the staining increased in fat body $2-12 \mathrm{~h}$ postinjection with 20E (h.p.i.20E) but became less intense at 24 h.p.i.20E (Fig. 1). The expression of the autophagyrelated genes, Atg1 and Atg8, in the fat body at 2, 6, 12, and 24 h.p.i.20E showed that their expression did not change at 2 h.p.i.20E, but had increased significantly at 6 and 12 h.p.i.20E, then decreased at 24 h.p.i.20E (Fig. 2).

Based on these results, and those of previous studies [10, 42], 12 libraries from controls and treated fat bodies at 2 and 6 h.p.i.20E were constructed and sequenced. Approximately 82 to 108 million raw reads were generated per sample, and 80 to 105 million clean reads per sample with high quality were retained. Approximately $85.12-90.61 \%$ of clean reads were mapped to the silkworm genome, and $75.91-85.27 \%$ of clean reads were uniquely mapped. The clean reads were mapped with a reference annotation, and 56.67-64.67\% of them were mapped to mRNAs (Additional file 1: Table S1).

The putative lncRNAs were identified following several filtering steps (Fig. 3a). The protein-coding potential of each transcript was predicted using the Coding Potential Calculator (CPC, http://cpc.gao-lab.org/) and the Protein Families Database (PFAM) [43] (Fig. 3b). In total, 1035 putative lncRNAs were identified. According to their genomic location and neighboring genes, they were classified into two types: lincRNA (long intergenic noncoding RNA) and antisense lncRNA. LincRNAs are transcripts located in the intergenic regions between two protein-coding genes. Antisense lncRNAs are transcripts that have exonic overlap with a known protein-coding gene on the opposite strand. A total of $87.3 \%$ of the identified lncRNAs were lincRNAs, and $12.7 \%$ were antisense lncRNAs (Fig. 3c). Information about all lncRNAs is shown in Additional file 2 (Table S2).

\section{Characteristic features of IncRNAs and mRNAs}

A total of 1035 lncRNAs and 14,622 mRNAs were obtained from the fat body of silkworm (Additional file 3: Table S3). The features of the lncRNAs, including transcript length, exon number, and expression levels, were assessed and compared with those of mRNAs. The size of the lncRNAs varied from 212 nt to 42,442 nt, with $60 \%$ of lncRNAs having a length $\geq 1000$ nt. The mean length of lincRNAs was $2412 \mathrm{nt}$, and that of antisense IncRNAs was $4027 \mathrm{nt}$, greater than the mean length of mRNAs (1224 nt) (Fig. 4a). LncRNAs had fewer exons than mRNAs: 2.48 for lincRNAs and 2.95 for antisense lncRNAs versus 5.44 for mRNAs on average (Fig. 4b), and the expression level of lncRNAs was lower than that of mRNAs (Fig. 4c). These results provided an overview of transcriptional changes in the expression of lncRNAs in the silkworm fat body in response to $20 \mathrm{E}$ treatment.

\section{Differential expression of IncRNAs and mRNAs}

Expression changes of lncRNAs and mRNAs in different silkworm samples were investigated based on the Fragments Per Kilobase of exon model per Million mapped fragments (FPKM) values of genes. In total, 166 differentially expressed lncRNAs and 3041 mRNAs were detected in silkworm fat body after 20E injection (Fig. 5). Thirty-five upregulated and 97 downregulated lncRNAs

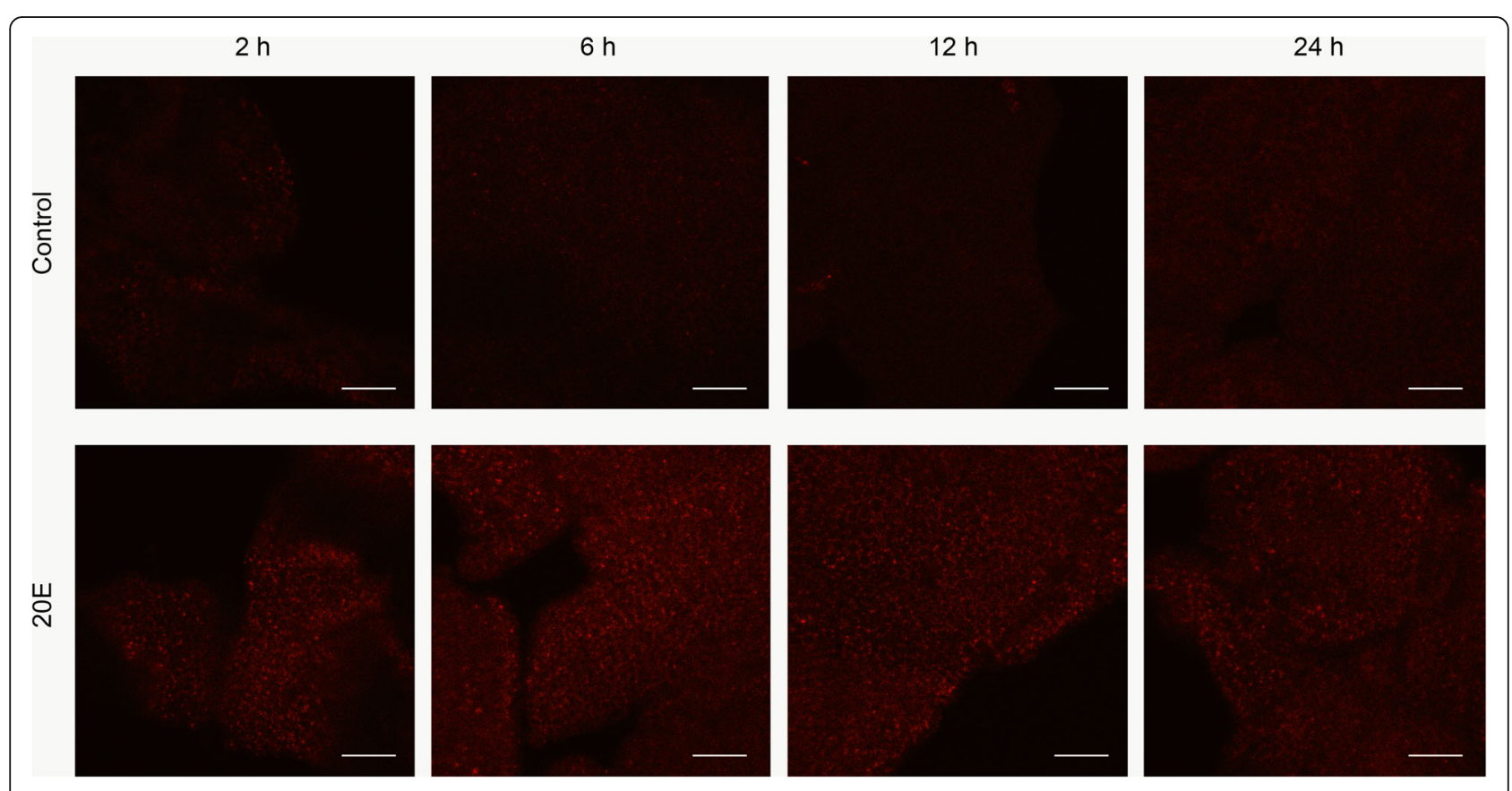

Fig. 1 Autophagy detection after 20E treatments in B. mori fat body by LysoTracker Red staining (red, magnification $40 \mathrm{x}$, the scale is $50 \mu \mathrm{m}$ ) 

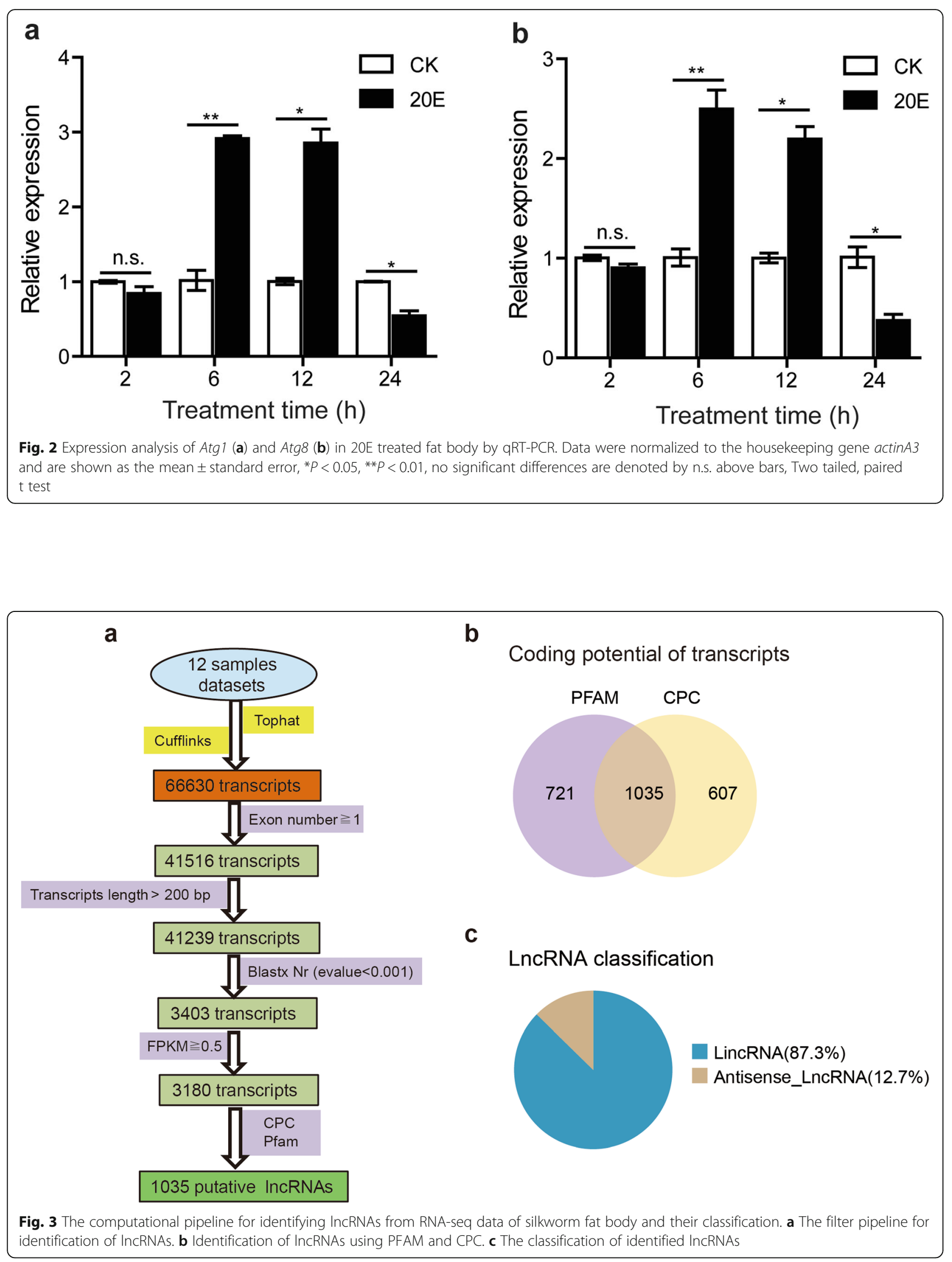

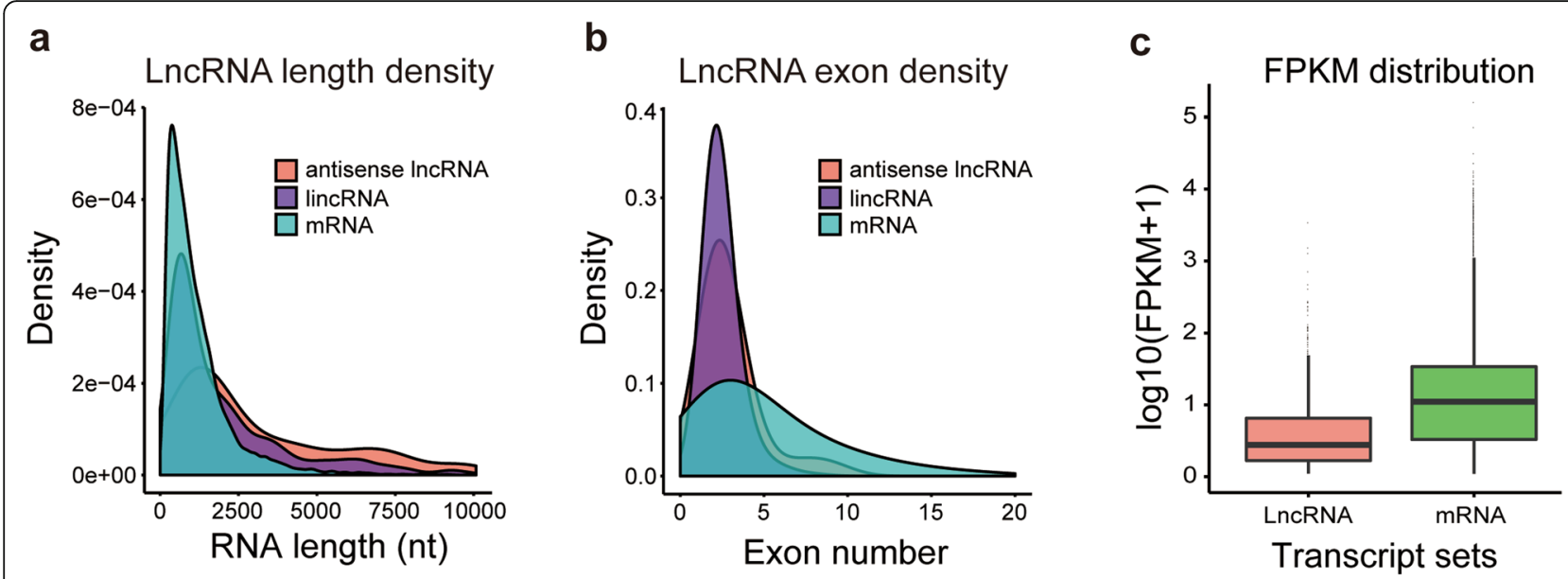

Fig. 4 Features of silkworm IncRNAs and mRNAs. a Transcript size distribution of lincRNAs, antisense IncRNAs, and mRNAs. b Number of exons per transcript of lincRNAs, antisense IncRNAs, and mRNAs. c Expression level indicated by $\log 10(\mathrm{FPKM}+1)$ in the IncRNAs and mRNAs

were found at 2 h.p.i.20E between treated and control (T_2h vs. C_2h), 31 upregulated and 33 downregulated lncRNAs were found at 6 h.p.i.20E between treated and control (T_6h vs. C_6h), 878 upregulated and 1177 downregulated mRNAs were detected in the T_2h vs. C_2h group, and 755 upregulated and 972 downregulated mRNAs in the T_6h vs. C_6h group (Table 1). As shown in Figs. 5 and 30 differentially expressed lncRNAs and 741 differentially expressed mRNAs were shared between the two groups (Additional file 4: Table S4). Heatmaps constructed from these data are shown in Fig. 6.

\section{GO and KEGG analysis of IncRNA target genes}

To explore the function of the identified lncRNAs, their potential target genes were predicted using cis (co-location) and trans (co-expression) methods. A total of 6493 cisregulatory IncRNA-mRNA pairs were predicted within a region $100 \mathrm{~kb}$ upstream and downstream of lncRNAs, of which 1032 were within $10 \mathrm{~kb}$ upstream and downstream of the nearby target genes. GO analysis [44] showed that four GO terms were significantly enriched (corrected $p$ value $<0.05)$ in the T_2h vs. C_2h group. However, there was no significant enrichment of GO terms in the T_6h vs. C_6h group (Additional file 5: Table S5). KEGG analysis [45] indicated that 94 pathways were enriched in cis-regulatory target genes of lncRNAs in the two groups. The most enriched pathways included "Phosphatidylinositol signaling system," "Ribosome biogenesis," and "Glyoxylate and dicarboxylate metabolism." The pathways "Hippo signaling pathway - fly," "Notch signaling pathway," and "Wnt signaling pathway" were common among the top 20 enriched pathways (Additional file 6: Table S6).

With respect to the trans regulation of lncRNAs, 42,797 trans-acting lncRNA-mRNA pairs were predicted, including 42,651 positive correlation IncRNA-mRNA pairs $(r>$ $0.95)$ and 146 negative correlation pairs $(r<-0.95)$. There

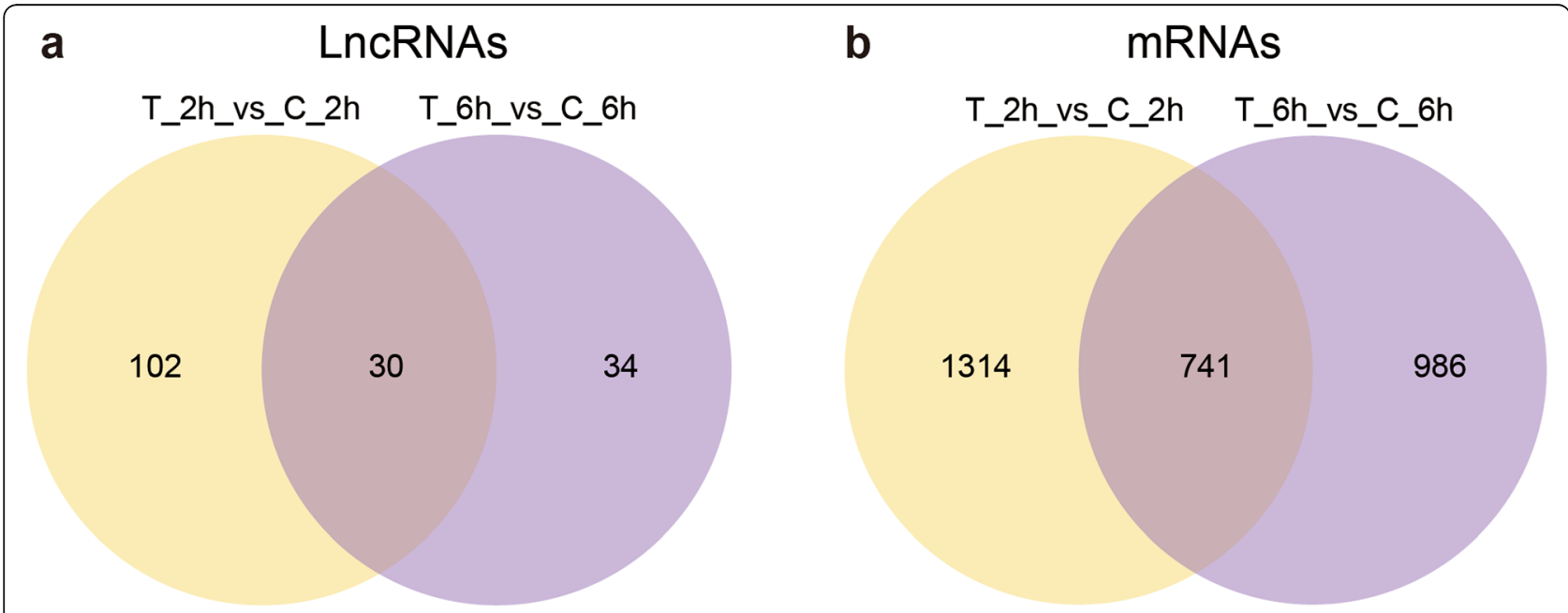

Fig. 5 Overlapped differentially expressed IncRNAs (a) and mRNAs (b) in T_2h vs. C_2h and T_6h vs. C_6h 
Table 1 Number of differentially expressed transcripts in each group

\begin{tabular}{llcc}
\hline Transcripts & & T_2h vs. C_2h & T_6h vs. C_6h \\
\hline IncRNA & up-regulated & 35 & 31 \\
& down-regulated & 97 & 33 \\
\multirow{2}{*}{ mRNA } & up-regulated & 878 & 755 \\
& down-regulated & 1177 & 972 \\
\hline
\end{tabular}

were 45 significantly enriched GO terms (corrected $p$ value $<0.05$ ) in the T_2h vs. $C_{-} 2 h$ group, and none in the T_6h vs. C_6h group. Twenty GO terms associated with metabolism or biosynthesis were enriched in the category Biological Process, two in Cell Component and two in Molecular Function (Additional file 7: Table S7). KEGG analysis showed 98 pathways enriched in trans-acting target genes of IncRNAs in the two groups. The most enriched pathways were "Fatty acid biosynthesis," "Citrate cycle," and "Proteasome." In the two groups, "Fatty acid biosynthesis," "Fatty acid metabolism," and "Citrate cycle" were the most enriched pathways among the downregulated target genes, while "Proteasome" and "Lysosome" were the most enriched pathways in the upregulated target genes. "Wnt signaling pathway" was the most common pathway among the top 20 enriched pathways in the T_6h vs. C_6h group (Additional file 8: Table S8).

\section{Functional analysis of mRNA in silkworm fat body}

Differentially expressed mRNAs were analyzed using GO and KEGG enrichment. Seven GO terms were significantly enriched in the Biological Process and Molecular Function categories in the T_2h vs. C_2h group. Sixteen GO terms were significantly enriched in the T_6h vs. C_6h group (corrected $p$-value $<0.05$ ), including 9 in Biological Process, 3 in Cell Component, associated with proteasome complex, and 5 in Molecular Function, related to different enzyme activities (Additional file 9: Table S9). KEGG analysis showed 113 pathways enriched in the differentially expressed mRNAs in the two groups. The most enriched pathways of mRNAs in the two groups were "Citrate cycle," "Fatty acid metabolism," and "Proteasome." "Fatty acid metabolism," "Fatty acid biosynthesis," and "Citrate cycle" were the most enriched pathways for downregulated genes, and "Proteasome," "Lysosome," and "Peroxisome" were the most enriched pathways for upregulated genes. "Regulation of autophagy," "Hippo signaling pathway-fly," "Notch signaling pathway," and "Jak-STAT signaling pathway" were the most common pathways in both groups (Additional file 10: Table S10).
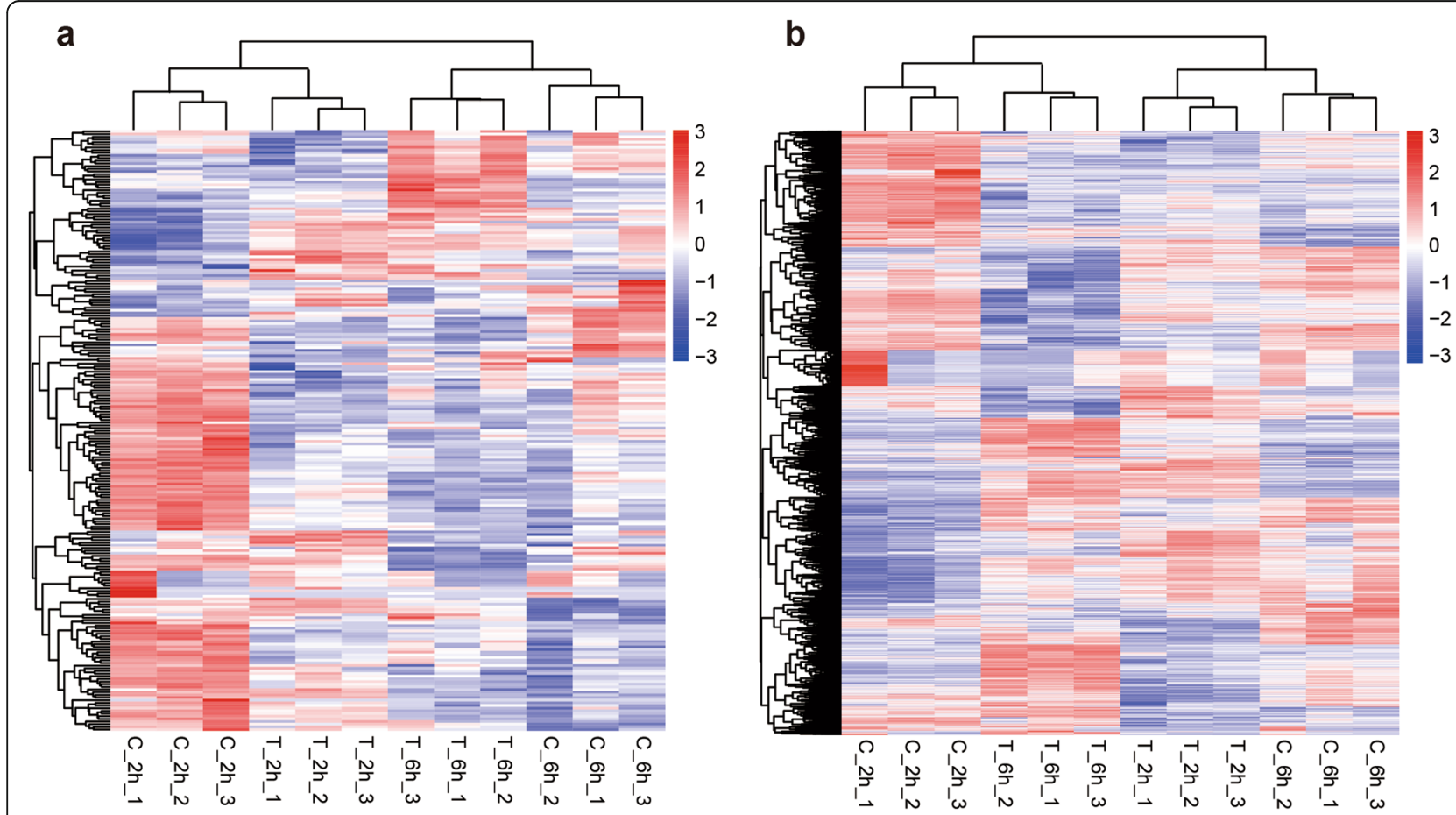

Fig. 6 Hierarchical clustering of the differentially expressed IncRNAs (a) and mRNAs (b) in T_2h vs. C_2h and T_6h vs. C_6h 
Validation and detection of differentially expressed IncRNAs and mRNAs

To validate the RNA-seq results, four lncRNAs and two mRNAs were chosen for qRT-PCR analysis. $L N C_{-}$ 000560 and $L N C \_000063$ were significantly upregulated at 2 and 6 h.p.i.20E compared with control, whereas LNC_000458 and LNC_000585 were significantly downregulated. The predicted trans target genes $(r>0.95)$ of
LNC_000560 and LNC_000063 were Atg4B and HR3 respectively, which are essential proteins playing important roles in 20E regulated autophagy and the molting or metamorphosis of insects. As shown in Fig. 7, $L N C_{-}$ 000560, LNC_000063, LNC_000458, LNC_000585, Atg4B, and HR3 showed significantly different expression at 2 and 6 h.p.i.20E, and observation was consistent with the RNA-seq data.
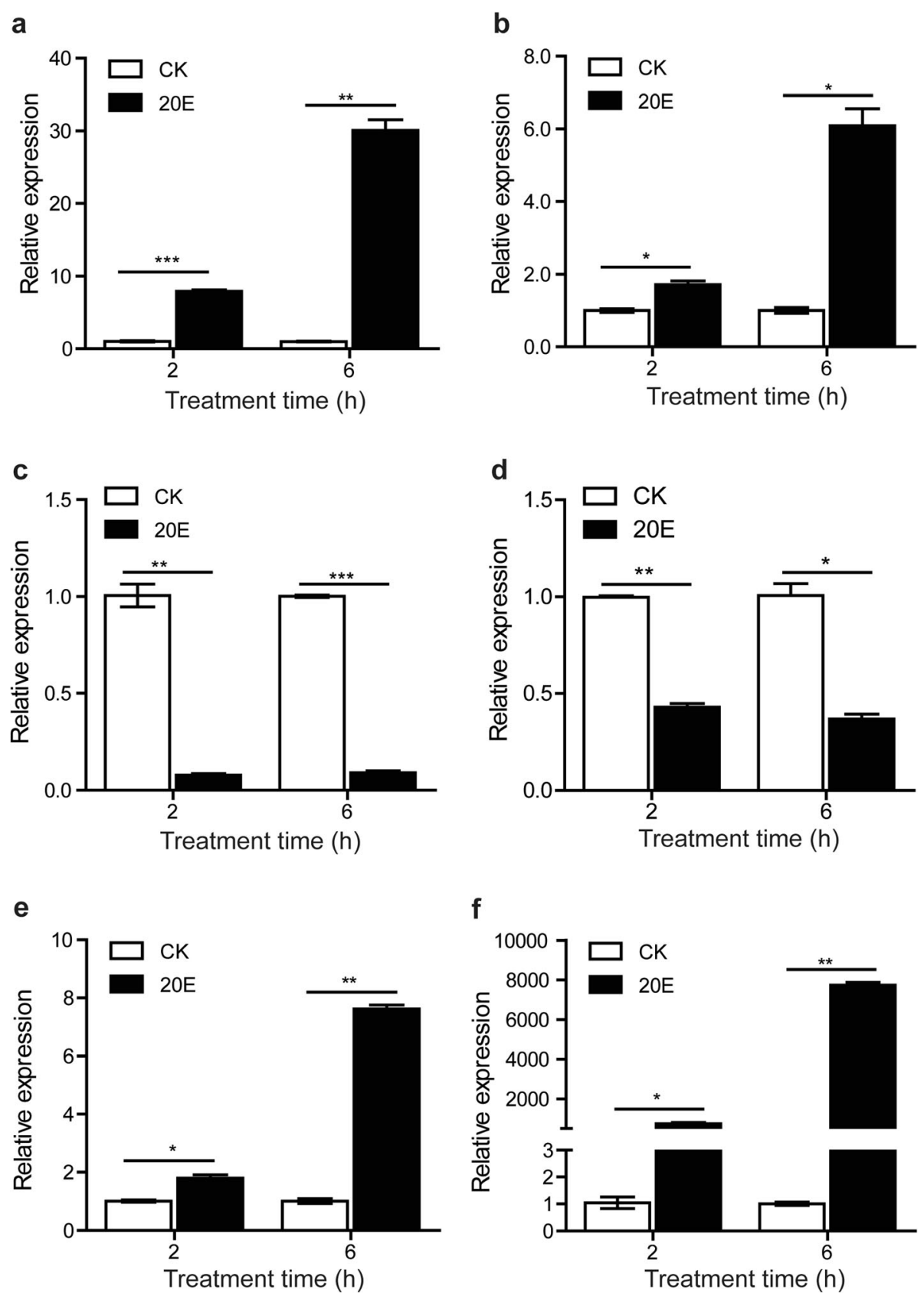

Fig. 7 qRT-PCR validation of selected InCRNAs and mRNAs in 20E treated fat body. a LNC_000560, b LNC_000063, c LNC_000458, d LNC_000585, e Atg4B, f HR3. Data were normalized to the housekeeping gene actinA3 and are shown as the mean \pm standard error, ${ }^{*} P<0.05,{ }^{*} P<0.01,{ }^{* * *} P<$ 0.001 , Two tailed, paired t test 
Because Atg4B has not been functionally identified in B. mori, the phylogenic analysis of ATG4B homologs from different species was performed. The results showed that Atg4B of B. mori was more conserved with the homologs from other lepidopteran species and $D$. melanogaster Atg4A. The sequence identitiy was $100 \%$ identical to the Atg4B of B. mandarina, $88 \%$ to Helicoverpa armigera and Manduca sexta Atg4B, $50 \%$ to the D. melanogaster Atg4A, $47 \%$ to Homo sapiens ATG4B, and $46 \%$ to Mus musculus ATG4B, but only $33 \%$ identities to the B. mori Atg4-like, $30 \%$ to the Drosophila Atg4B, and $25 \%$ to the Saccharomyces cerevisiae Atg4 (Fig. 8). The alignment of the sequences showed that the predicted functional domain, peptidase C54, was evolutionary conservation among the different ATG4 homologs (Fig. 9).

Since Atg4B is an essential protein involved in autophagy, to further study the function of lncRNA in 20Einduced autophagy, we focused on LNC_000560 and its target gene $\operatorname{Atg} 4 B$ to explore the regulatory function of lncRNAs in the silkworm. We further analyzed the expression profiles of $L N C \_000560$ and Atg $4 B$ in the fat body at 2, 6, 12, and 24 h.p.i.20E, at different developmental stages and in different tissues of silkworm larvae. $L N C_{-}$ 000560 and $A \operatorname{tg} 4 B$ showed highly similar expression patterns in the fat body. The expression of $L N C \_000560$ and Atg $4 B$ was significantly increased at 2, 6, and 12 h.p.i.20E, and decreased to basal levels at 24 h.p.i.20E (Fig. 10).

Their expression trend at different developmental stages was also consistent, while several peaks were detected from the second day of 5th instar larvae to the adult stage. Especially both exhibited expression peaks at the later stage of larvae and pupae, as well as on the second day of the pupal stage (Fig. 11a). The expression of LNC_000560 in larval tissues was high in the epidermis and fat body, and relatively low in other tissues, with no expression in the hemolymph or midgut (Fig. 11b). Atg $4 B$ showed a similar expression pattern as $L N C_{-}$ 000560, although its highest expression was observed in testis (Fig. 11c).

\section{RNA interference of IncRNA}

To further study the regulation relation between $L N C_{-}$ 000560 and $A \operatorname{tg} 4 B$, we performed RNAi experiment to knockdown the expression of LNC_000560 in the fat body of silkworm larvae. Compared with dsEGFP control, we found that the expression of $L N C \_000560$ and Atg $4 B$ in fat body was significantly decreased after LNC_ 000560 knockdown, which indicated that the expression of LNC_000560 was successfully down-regulated and it could indirectly participate in the regulation of autophagy by regulating its target gene $\operatorname{Atg} 4 B$ (Fig. 12).

\section{Discussion}

In insects, the fat body undergoes dramatic changes, via a process known as fat body remodeling, during metamorphosis from larva to pupa [34, 46, 47]. Fat body remodeling is regulated mainly by $20 \mathrm{E}$ and the AMPKTOR pathway [48]. To identify lncRNAs involved in 20E-induced autophagy in the fat body, we chose the 2 and 6 h.p.i.20E experimental times for RNA-seq analysis. A total of 1035 putative lncRNAs were obtained, including 905 lincRNAs and 130 antisense lncRNAs. Previous studies have identified 11,810 lncRNAs from 21 different

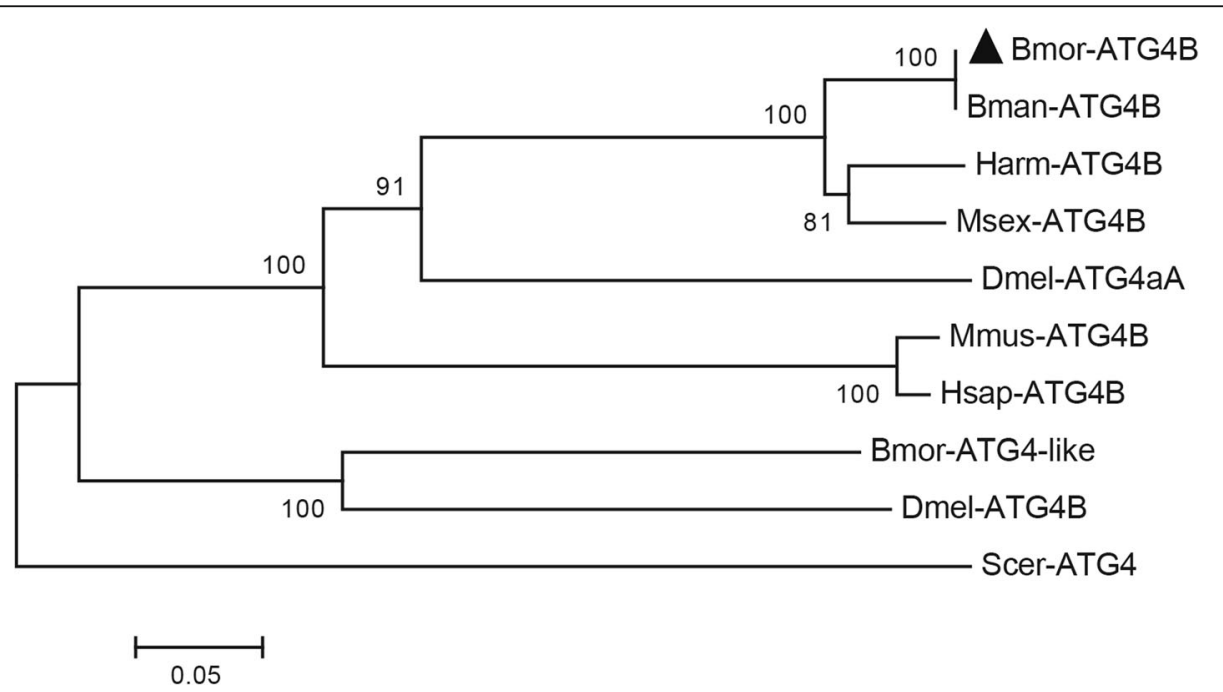

Fig. 8 Phylogenetic analysis of the ATG4 homologs from different species. Bmor: Bombyx mori (Atg4B: XP_004929228.2; Atg4-like: ACJ46060.1), Bman: Bombyx mandarina (XP_028029080.1), Harm: Helicoverpa armigera (XP_021182852.1), Msex: Manduca sexta (XP_030033081.1), Dmel: Drosophila melanogaster (Atg4A: NP_608563.1; Atg4B: NP_650452.1), Mmus: Mus musculus (NP_777363.1), Hsap: Homo sapiens (AAH00719.1), Scer: Saccharomyces cerevisiae (NP_014176.2). 

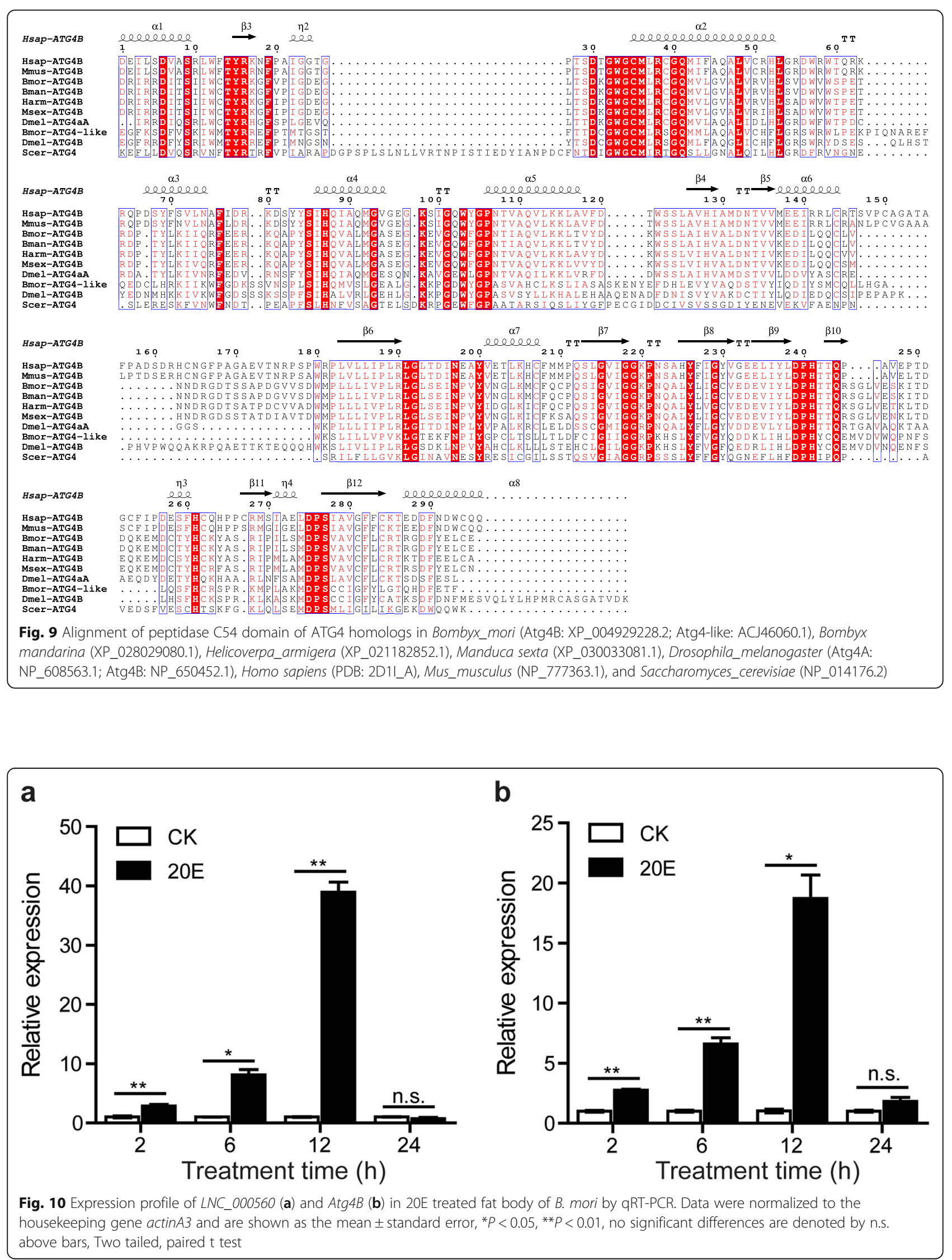

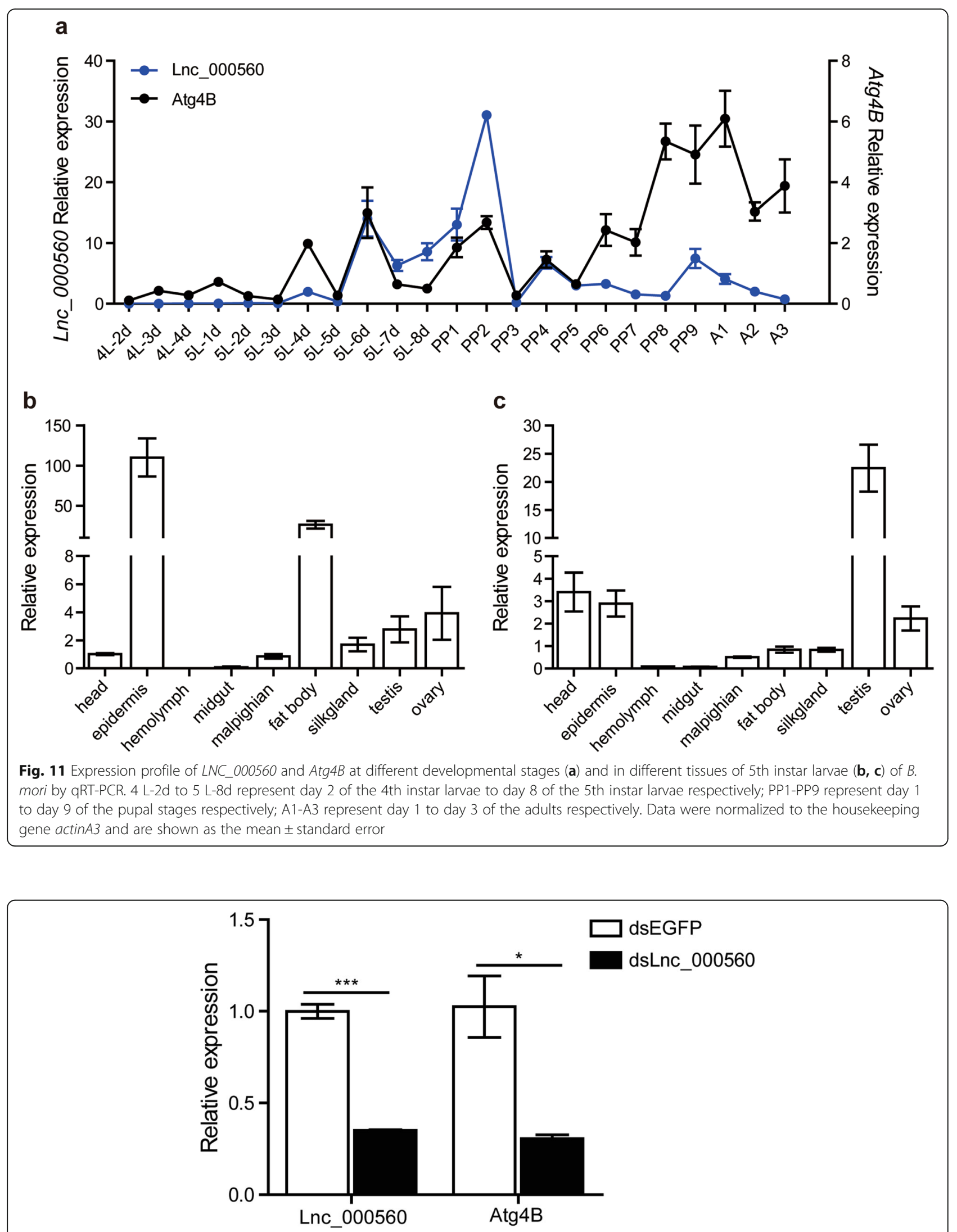

Fig. 12 Expression of LNC_000560 and Atg4B after RNAi of LNC_000560 in fat body of 5th instar larvae of B. mori by qRT-PCR 
tissues [36], 6281 lncRNAs from the available RNA-seq data of B. mori [37], 599 lncRNAs in the silk gland [38], 13,159 lncRNAs in BmN cells [41]. The divergence of lncRNAs numbers in $B$. mori may be due to the use of different samples and methods. Most of the lncRNAs were located in intergenic regions, and had 2-3 exons, which were consistent with $65.6 \%$ lincRNA in $\mathrm{BmN}$ cell and $74.83 \%$ in silk gland. LncRNAs, especially antisense lncRNA, were longer than mRNAs, although they possessed a lower number of exons, which were also similar to those observed in previous studies [36-38]. Silkworm lncRNAs showed lower expression levels than proteincoding mRNAs observed, as in mammals [49].

Differential expression analysis showed that 166 differentially expressed lncRNAs and 3041 mRNAs appeared to be related to the 20E response, among which 30 differentially expressed lncRNAs and 741 such mRNAs were shared between the two groups (T_2h vs. $C_{-} 2 h$ and T_6h vs. C_6h). The cis and trans method was used to predicted the function of the identified lncRNAs, we found 6493 lncRNA-mRNA pairs in the cis prediction and 42,797 lncRNA-mRNA pairs in the trans prediction. KEGG analysis showed that most of the pathways enriched in cis-regulatory genes were different from those enriched in trans-acting genes. The pathways most enriched in differentially expressed mRNAs were "Citrate cycle," "Fatty acid metabolism," and "Proteasome," similar to the trans-acting target genes. Therefore, we speculated that the identified lncRNAs might function mainly as trans-acting genes rather than as cis-regulatory genes. Besides, among 42,797 trans-acting lncRNAmRNA pairs, more than $99 \%$ of them were positive correlation. The pathways most enriched in both downregulated trans-acting target genes and mRNAs were "Fatty acid biosynthesis," "Fatty acid metabolism," and "Citrate cycle," whereas, those enriched in upregulated genes were "Proteasome" and "Lysosome." The insect fat body is the main organ involved in energy metabolism and is analogous to the adipose tissue and liver in vertebrates. In Drosophila, the fat body is a crucial tissue controlling energy storage and utilization, and meeting the changing energy demands of all developmental stages. Lipids, composed of fatty acids and cholesterol, are the main form of energy storage [47]. In the last decade, several studies have found that the expression of many genes involved in energy metabolism is downregulated by $20 \mathrm{E}$ in the Drosophila midgut and fat body, blocking their metabolic activity for the initiation of metamorphosis [50, 51]. Further evidence in silkworm indicated that the fat body is a responsive tissue, which modifies metabolic activity in response to molting and pupation induced by 20E [52]. Autophagy-lysosome and ubiquitin-proteasome are the two major protein degradation systems in all eukaryotic cells. They are responsible for maintaining protein homeostasis by degrading intracellular proteins [53]. Hence, we assume that the identified lncRNAs may function mainly through the positive correlation target genes, and the downregulation of these genes may play a role in fat body development, and upregulated genes may function mainly in the degradation of intracellular proteins via autophagy or ubiquitin pathway.

Several IncRNAs have been identified in the silkworm, but only a few of them have so far been functionally characterized [35, 39, 40]. We predicted 71 trans-regulated target genes for $L N C_{-} 000560,8$ for $L N C_{-} 000063$, 12 for $L N C \_000458$, and 50 for $L N C$ _000585. We selected $L N C \_000560$ and $L N C \_000063$ to verify the expression of their target genes in the fat body. The results showed that $A \operatorname{tg} 4 B$ and $H R 3$ were significantly upregulated, similar to $L N C \_000560$ and $L N C \_000063$.

Atg4B, a target gene of $L N C_{-} 000560$, is the main human ortholog of four different ATG4 family members involved in autophagy and can cleave most of the human ATG8 homologs [54]. Increases in ATG4B levels are accompanied by the induction of autophagy in pneumonia and fibrosis [55]. The phylogenic analysis and functional domain alignment indicated that, apart from the Atg4B of lepidopteran species, the B. mori Atg4B was more conserved with $D$. melanogaster Atg4A and mammals ATG4B, and B. mori Atg4B-like was more conserved with $D$. melanogaster Atg4B. The functional difference between Atg4B and Atg4B-like in B. mori requires more investigation. In $B$. mori, a previous study demonstrated that Atg4-like is the least sensitive to 20E of the $13 \mathrm{Atg}$ genes [10]. In our study, Atg $4 B$ was significantly upregulated after $20 \mathrm{E}$ treatment, which indicated that Atg $4 B$ expression was more sensitive to 20E than Atg4like. The expression profiles of LNC_000560 and Atg4B were highly consistent in 20E-treated fat bodies and in the different developmental stages from larvae to adults. Moreover, RNAi of LNC_000560 significantly decreased the mRNA level of Atg4B. While autophagic activity is reduced in Atg4 deficient yeast and Atg $4 b$ deficient mice, the deletion of $\operatorname{Atg} 4 \mathrm{c}$ in mice has little impact on autophagy, Atg4d silencing prevents autophagosome formation and induces cell death [54-56]. Therefore, $L N C_{-}$ 000560 may be involved in the 20E-induced autophagy of the fat body by up-regulating its target gene $A \operatorname{tg} 4 B$. However, it remains unclear how $L N C$ _000560 regulate the expression of its target genes and participate in autophagy and the development of B.mori. Further studies should be conducted to clarify the regulatory mechanism between lncRNAs and their target gene.

\section{Conclusions}

In the current study, 1035 lncRNAs were identified from 12 RNA-seq libraries of the B. mori fat body, including 
905 lincRNAs and 130 antisense lncRNAs. In total, 166 lncRNAs showed significant changes in expression when responding to 20E. Prediction of target genes showed that some lncRNAs and mRNAs were involved in important biological processes, such as autophagy and molting. The functional study of $L N C_{-} 000560$ and its target genes indicated a potential role of $L N C \_000560$ in 20E-induced autophagy of $B$. mori via the indirect regulation of Atg4B. These results provide a solid foundation for further study into the regulatory mechanisms of IncRNAs in 20E-induced autophagy, and their roles in other biological processes in B. mori.

\section{Methods}

\section{Silkworm rearing and tissue collection}

All experiments were carried out with silkworm strain Dazao p50, obtained from the Sericultural Research Institute, Chinese Academy of Agricultural Sciences. Larvae were reared on fresh mulberry leaves at $25^{\circ} \mathrm{C}$ under $12 \mathrm{~h}$ light/ $12 \mathrm{~h}$ dark cycles.

The 20E levels in the 2-day-old 5th instar larvae are low, and the fat body is sensitive to 20E [57]. Accordingly, insects at this stage of development were chosen for injection of $5 \mu \mathrm{g} 20 \mathrm{E}$ (Solarbio, SE8010) per larva. Control insects were injected with the same volume of solvent. The fat body tissues of ten individuals were dissected from the larval abdominal segment at 2, 6, 12, and 24 h.p.i.20E, and tissues from each time point were pooled. The insects' whole bodies from each day of the 4th and 5th instar stages after discarding the food residues, as well as each day of pupae until the 3rd day of adulthood were collected. The head, epidermis, hemolymph, midgut, malpighian tubules, fat body, silk gland, testis, and ovary were collected from the 3rd day of 5th instar larvae. Three biological replicates were used for each experiment. All samples were frozen immediately in liquid nitrogen and stored at $-80^{\circ} \mathrm{C}$ until use.

\section{LysoTracker Red staining}

LysoTracker is an acidotropic dye that stains cellular acidic compartments, including lysosomes and autolysosomes. It has been used to detect autophagy-associated lysosomal activity from yeast to human [58, 59]. LysoTracker Red staining has proven to be an effective indicator of autophagy in B. mori fat body [10]. Newly collected fat body tissues were fragmented by forceps, washed with PBS, stained with LysoTracker Red DND99 at a final concentration of $50 \mathrm{nM}$ (L7528, Thermo Fisher Scientific, USA) for $5 \mathrm{~min}$ at $37^{\circ} \mathrm{C}$, and washed again with PBS 3 times. The LysoTracker Red staining of the samples was observed under a Nikon Eclipse C1 confocal microscope.
RNA extraction, library construction and sequencing Total RNA from the silkworm's fat body tissue at 2 and 6 h.p.i.20E was extracted using the Trizol reagent (Invitrogen) and further purified with RNeasy kits (Qiagen). RNA quantification and quantification were detected in Novogene Bioinformatics Institute (Beijing, China) following standard procedure. RNA purity was checked using a NanoPhotometer spectrophotometer (IMPLEN, CA, USA). RNA concentration was measured using Qubit RNA Assay Kits in a Qubit 2.0 Flurometer (Life Technologies, CA, USA). RNA integrity was assessed using RNA Nano6000 Assay Kits from the Bioanalyzer 2100 system (Agilent Technologies, CA, USA).

RNA-seq was carried out following standard Illumina protocols as previous reported [60]. A total of $3 \mu \mathrm{g}$ RNA per sample was used as input material for RNA sample preparation. Ribosomal RNA was removed using Epicentre Ribo-zero $^{\text {tm }}$ rRNA Removal Kits (Epicentre, USA), and rRNA-free residue was cleaned up by ethanol precipitation. Subsequently, sequencing libraries were generated using the rRNA-depleted RNA with NEBNext Ultra ${ }^{\text {me }}$ Directional RNA Library Prep Kits for Illumina (NEB, USA), following manufacturer's recommendations. Finally, the products were purified, and the library quality assessed using the Agilent Bioanalyzer 2100 system. The clustering of the index-coded samples was performed on a cBot Cluster Generation System using TruSeq PE Cluster Kits v3-cBot-HS (Illumina), according to the manufacturer's instructions. After cluster generation, the libraries were sequenced on an Illumina HiSeq 2500 platform (Novogene Bioinformatics Institute, Beijing, China) and 125 bp paired-end reads were generated.

\section{Mapping to reference genome}

Data analysis was conducted by the Novogene Gene Regulation Department (Beijing, China) as previous reported [60]. Raw read data in FASTQ format were processed using custom Perl scripts. In this step, clean data were obtained by removing reads containing adapter or ploy- $\mathrm{N}$, and low-quality reads, from the raw data. The Q20, Q30, and GC contents of the clean reads were calculated. All downstream analysis were based on the clean, high quality reads. Reference genome and gene annotation files of silkworm $B$. mori were downloaded from SilkDB website (http://silkworm.genomics.org.cn/) and NCBI genome site (https://www.ncbi.nlm.nih.gov/ genome/). An index of the reference genome was built using Bowtie v2.0.6 and paired-end clean reads were aligned to the reference genome using TopHat v2.0.9 (http://ccb.jhu.edu/software/tophat/index.shtml) [61].

\section{Transcriptome assembly}

The mapped reads of each sample were assembled using both Scripture (beta2) [62] and Cufflinks v2.1.1 (http:// 
cole-trapnell-lab.github.io/cufflinks/) [63] in a referencebased approach. Both methods use spliced reads to determine exon connectivity, but the methods use two different approaches. Scripture uses a statistical segmentation model to distinguish expressed loci from experimental noise, and uses spliced reads to assemble expressed segments. It reports all statistically significantly expressed isoforms at each locus. Cufflinks uses a probabilistic model to simultaneously assemble and quantify the expression level of a minimal set of isoforms which provides a maximum likelihood explanation of the expression data in each locus.

\section{LncRNA identification and classification}

The putative lncRNAs were screened and identified from the silkworm transcriptome. The filtering process included five steps: (1) single exon transcripts were eliminated; (2) transcripts of $\leq 200 \mathrm{bp}$ were removed; (3) transcripts that overlapped with any protein-coding exon in the sense orientation were filtered out; (4) transcripts with fragments per kilobase of transcripts per million mapped reads $(\mathrm{FPKM})<0.5$ were removed; and (5) transcripts with protein-coding potential as predicted by either CPC or PFAM were excluded, while transcripts without protein-coding potential made up the candidate set of lncRNAs [64, 65]. The putative lncRNAs were classified into two groups, lincRNA and antisense lncRNA, using the class code module in Cuffcompare (http://coletrapnell-lab.github.io/cufflinks/cuffcompare/) [66].

\section{Expression analysis of IncRNAs and mRNAs}

The expression levels of both lncRNAs and proteincoding genes in each sample were measured using Cuffdiff (v2.1.1) [66]. Gene FPKMs were calculated by summing the FPKMs of transcripts in each gene group. FPKM stands for fragments per kilobase of exon per million mapped fragments, which is calculated based on the length of the fragments and reads count mapped to each fragment. Cuffdiff provides statistical routines for determining differential expression in digital transcript or gene expression data, using a model based on the negative binomial distribution. Transcripts with a $q$ value $<0.05$ were identified as differentially expressed.

\section{Co-location analysis and functional enrichment}

Cis-regulatory lncRNAs target and regulate the neighboring protein-coding genes $[67,68]$. We searched the protein-coding genes within $100 \mathrm{~kb} / 10 \mathrm{~kb}$ upstream or downstream of all the identified lncRNAs, and then analyzed their function by functional enrichment. All colocated genes were used for GO enrichment analysis and KEGG enrichment analysis. GO enrichment analysis of the neighbor genes were implemented using goseq [69]. KEGG metabolic pathway enrichment analysis was carried out using KOBAS2.0 [70]. GO terms and KEGG pathways with $p$-values $<0.05$ were considered significantly enriched.

\section{Co-expression analysis}

The expression levels of the identified lncRNAs and mRNAs from the two different stages were used to analyze the co-expression relationships of lncRNAs and protein-coding genes. Pearson's correlation coefficients between the expression levels of 1035 lncRNAs and 14, 622 mRNAs were calculated with custom scripts ( $r>$ 0.95 or $\mathrm{r}<-0.95$ ), and the candidate lncRNA target genes were used for functional enrichment analysis to predict the function of IncRNAs. Hypergeometric test and Benjamini-Hochberg FDR (false discovery rate) correction were used for statistically analysis, and only GO terms or KEGG pathways with corrected $p$-values $<0.05$ or FDR $<0.05$ were considered significant.

\section{Expression validation of the selected IncRNAs and mRNAs} Atg1 is required for autophagosome initiation and is sensitive to 20E injection, Atg8 is the protein most widely used to monitor autophagy [71, 72]. The expression of the autophagy-related genes, Atg1 and Atg8, in the fat body at $2,6,12$, and 24 h.p.i.20E was evaluated using qRT-PCR. The relative expression levels of the differentially expressed lncRNAs was confirmed using qRT-PCR. Total RNA from silkworm fat body 2, 6, 12, and 24 h.p.i.20E, and different developmental stages and tissues were used for the first-strand cDNA synthesis using RevertAid First Strand cDNA Synthesis Kits (Thermo Fisher, USA), according to the manufacturer's instructions. qRT-PCR was carried out using Roche FastStart Universal SYBR Green Master (Rox) (Roche, Switzerland). Each reaction was performed on a BIORAD CFX96 Real-Time PCR Detection System with three biological replicates. The relative expression levels of lncRNAs were calculated using the $2^{-\Delta \Delta \mathrm{Ct}}$ method [73]. Silkworm actinA3 (GenBank accession number U49854) was used as a reference gene. All the primers were designed using Primer 5.0 software (Additional file 11: Table S11). The PCR products were sequenced by the Beijing Genomics Institute (BGI, Beijing).

\section{RNA interference}

The primers of LNC_000560 and EGFP containing the T7 promoter sequence were designed for RNA interference. Their double-stranded RNA (dsRNA) were synthesized using T7 RiboMAX ${ }^{\text {mi }}$ Express RNAi system (Promega, P1700) according to the manufacturer's instruction. The dsRNA (10 $\mu \mathrm{g}$ per larva) of $L N C \_000560$ was injected into 2-day-old 5th instar larvae, and EGFP dsRNA was injected as a control. After injection for $24 \mathrm{~h}$, the fat body was dissected for qRT-PCR analysis. 
All the primers used in this study are listed in Additional file 11 (Table S11).

\section{Bioinformatics analysis}

Phylogenetic analysis was performed using MEGA5.0 software and the neighbor-joining method. The alignment of the peptidase C54 domain of ATG4B homologs was performed using the programs MultAlin [74] and ESPript [75]. All sequences were obtained from the NCBI and SilkDB databases.

\section{Statistics analysis}

Statistical analysis were performed using Prism 5, figures were prepared using Prism 5 and Adobe Illustrator CS5. The experimental data were analyzed using two-tailed, paired $t$-tests, $" p<0.05,{ }^{* *} p<0.01,{ }^{* * * *} p<0.001$. The values are shown as mean \pm standard deviation of three independent experiments.

\begin{abstract}
Abbreviations
LncRNA: Long non-coding RNA; 20E: 20-hydroxyecdysone; Atg: Autophagy related; JH: Juvenile hormone; EcR: Ecdysone receptor; HR3: Hormone receptor 3; PI3K: Phosphatidylinositol 3-kinase; TORC: Target of rapamycin complex; h.p.i.: Hours post-injection; FPKM: Fragments Per Kilobase of exon model per Million mapped fragments; CPC: Coding Potential Calculator; PFAM: Protein Families Database; ORF: Open Reading Frame; GO: Gene Ontology; KEGG: Kyoto Encyclopedia of Genes and Genomes;

AMPK: Adenosine 5'-monophosphate (AMP)-activated protein kinase
\end{abstract}

\section{Supplementary Information}

The online version contains supplementary material available at https://doi. org/10.1186/s12864-021-07692-1.

Additional file 1. Summary of RNA-seq data of 12 silkworm samples.

Additional file 2. Detailed information of IncRNAs identified in this study.

Additional file 3. LncRNA and mRNA features.

Additional file 4. Common differentially expressed IncRNAs and mRNAs in the two groups.

Additional file $\mathbf{5}$. The significantly enriched $\mathrm{GO}$ terms detected in the two groups (cis).

Additional file 6. The top 20 enriched KEGG pathways in the two groups (cis).

Additional file 7. The significantly enriched $\mathrm{GO}$ terms detected in the two groups (trans).

Additional file 8. The top 20 enriched KEGG pathways in the two groups (trans).

Additional file 9. The significantly enriched $\mathrm{GO}$ terms of differentially expressed mRNAs in the two groups.

Additional file 10. The top 20 enriched KEGG pathways of differentially expressed mRNAs in the two groups.

Additional file 11. The primers of the selected IncRNAs and mRNAs.

\section{Acknowledgements}

We thank Beijing Novogene Bioinformatics Institute, Beijing, China for assisting in sequencing and bioinformatic analysis. We also thank Muwang Li for providing the Bombyx mori strains from the Sericultural Research Institute, Chinese Academy of Agricultural Sciences, and Camilo AYRA-PARDO for reviewing the manuscript and his editorial assistance.

\section{Authors' contributions}

YK conceived the idea and supervised this research. HQ designed the study, performed data analysis and wrote the manuscript. YK and HQ obtained the research funds. HQ, JW and YW detected and collected samples for the library construction. JW and JY performed RNA extraction and expression analysis of the identified IncRNAs. BFW, ML, BW, and XL participated in rearing, injection and sampling of B. mori. YC and LT provided technical assistance and coordination. DL and LY assisted in the data analysis and revision. All authors read and approved the final manuscript.

\section{Funding}

This work was supported by grants from the National Natural Science Foundation of China (No. 31201754 and 32070503). The funding bodies had no role in the design of the study and collection, analysis, and interpretation of data and in writing the manuscript.

\section{Availability of data and materials}

The datasets generated during the current study are available in the SRA database of the NCBI system with accession number of PRJNA672230 (https:// dataview.ncbi.nIm.nih.gov/object/PRJNA672230?reviewer=um7or07tegs52 t8kec6smbtne5).

\section{Declarations}

Ethics approval and consent to participate Not applicable.

\section{Consent for publication}

Not applicable.

\section{Competing interests}

The authors declare that they have no competing interests.

\section{Author details}

${ }^{1}$ China-UK-NYNU-RRes Joint Laboratory of insect biology, Henan Key Laboratory of Insect Biology in Funiu Mountain, Nanyang Normal University, 473061 Nanyang, Henan, China. ${ }^{2}$ School of Life Science, Zhengzhou University, 450001 Zhengzhou, Henan, China. ${ }^{3}$ State Key Laboratory of Silkworm Genome Biology / Biological Science Research Center, Southwest University, 400716 Chongqing, China. ${ }^{4}$ Guangdong Laboratory for Lingnan Modern Agriculture/Guangdong Provincial Key Laboratory of Agro-animal Genomics and Molecular Breeding, College of Animal Science, South China Agricultural University, 510642 Guangzhou, Guangdong, China.

Received: 9 February 2021 Accepted: 6 May 2021

Published online: 22 May 2021

\section{References}

1. Klionsky DJ, Abdelmohsen K, Abe A, Abedin MJ, Abeliovich H, Acevedo Arozena $A$, et al. Guidelines for the use and interpretation of assays for monitoring autophagy (3rd edition). Autophagy. 2016;12(1), 1-222.

2. He C, Klionsky DJ. Regulation mechanisms and signaling pathways of autophagy. Annu Rev Genet. 2009;43:67-93.

3. Shintani T, Klionsky DJ. Autophagy in health and disease: a double-edged sword. Science. 2004;306(5698):990-5.

4. Levine B, Kroemer G. Biological Functions of Autophagy Genes: A Disease Perspective. Cell. 2019;176(1-2):11-42.

5. Mizushima N, Yoshimori T, Ohsumi Y. The role of Atg proteins in autophagosome formation. Annu Rev Cell Dev Biol. 2011;27:107-32.

6. Abreu S, Kriegenburg F, Gómez-Sánchez R, Mari M, Sánchez-Wandelmer J, Skytte Rasmussen M, et al. Conserved Atg8 recognition sites mediate Atg4 association with autophagosomal membranes and Atg8 deconjugation. EMBO Rep. 2017;18(5):765-80.

7. Li M, Hou Y, Wang J, Chen X, Shao ZM, Yin XM. Kinetics comparisons of mammalian Atg4 homologues indicate selective preferences toward diverse Atg8 substrates. J Biol Chem. 2011;286(9):7327-38.

8. Fernández ÁF, López-Otín C. The functional and pathologic relevance of autophagy proteases. J Clin Invest. 2015;125(1):33-41.

9. Zhang X, Hu ZY, Li WF, Li QR, Deng XJ, Yang WY, et al. Systematic cloning and analysis of autophagy-related genes from the silkworm Bombyx mori. BMC Mol Biol. 2009;10:50. 
10. Tian L, Ma L, Guo E, Deng X, Ma S, Xia Q, et al. 20-Hydroxyecdysone upregulates Atg genes to induce autophagy in the Bombyx fat body. Autophagy. 2013;9(8):1172-87.

11. Riddiford LM, Cherbas P, Truman JW. Ecdysone receptors and their biological actions. Vitam Horm. 2000;60:1-73.

12. Riddiford LM. Juvenile hormone action: a 2007 perspective. J Insect Physiol. 2008;54(6): 895-901.

13. Rusten $T E$, Lindmo $K$, Juhász $G$, Sass $M$, Seglen $P O$, Brech $A$, et al. Programmed autophagy in the Drosophila fat body is induced by ecdysone through regulation of the PI3K pathway. Dev Cell. 2004;7(2):179-92.

14. Sumithra $P$, Britto CP, Krishnan M. Modes of cell death in the pupal perivisceral fat body tissue of the silkworm Bombyx mori L. Cell Tissue Res. 2010;339(2):349-58.

15. Kapranov P, Cheng J, Dike S, Nix DA, Duttagupta R, Willingham AT, et al RNA maps reveal new RNA classes and a possible function for pervasive transcription. Science. 2007;316 (5830):1484-8.

16. Mercer TR, Dinger ME, Mattick JS. Long non-coding RNAs: insights into functions. Nat Rev Genet. 2009;10(3):155-9.

17. Young RS, Marques AC, Tibbit C, Haerty W, Bassett AR, Liu JL, Ponting CP. Identification and properties of 1,119 candidate lincRNA loci in the Drosophila melanogaster genome. Genome Biol Evol. 2012;4(4), 427-442.

18. Liu J, Jung C, Xu J, Wang H, Deng S, Bernad L, et al. Genome-wide analysis uncovers regulation of long Intergenic noncoding RNAs in Arabidopsis. Plant Cell. 2012;24(11):4333-4345.

19. Li L, Eichten SR, Shimizu R, Petsch K, Yeh CT, Wu W, et al. Genome-wide discovery and characterization of maize long non-coding RNAs. Genome Biol. 2014;15(2):R40.

20. Zhu B, Xu M, Shi H, Gao X, Liang P. Genome-wide identification of IncRNAs associated with chlorantraniliprole resistance in diamondback moth Plutella xylostella (L.). BMC Genomics. 2017;18(1):380.

21. Liu F, Shi T, Qi L, Su X, Wang D, Dong J, et al. LncRNA profile of Apis mellifera and its possible role in behavioural transition from nurses to foragers. BMC Genomics. 2019;20(1):393.

22. Chang ZX, Ajayi OE, Guo DY, Wu QF. Genome-wide characterization and developmental expression profiling of long non-coding RNAs in Sogatella furcifera. Insect Science, 2020;27(5):987-997.

23. Chen CK, Blanco M, Jackson C, Aznauryan E, Ollikainen N, Surka C, et al. Xist recruits the $X$ chromosome to the nuclear lamina to enable chromosomewide silencing. Science. 2016;354(6311):468-72.

24. Sahakyan A, Yang Y, Plath K. The Role of Xist in X-Chromosome Dosage Compensation. Trends Cell Biol. 2018:28(12):999-1013.

25. Han P, Chang CP. Long non-coding RNA and chromatin remodeling. RNA Biol. 2015;12(10):1094-1098.

26. Inoue A, Jiang L, Lu F, Zhang Y. Genomic imprinting of Xist by maternal H3K27me3. Genes Dev. 2017;31(19):1927-32.

27. Li K, Tian Y, Yuan Y, Fan X, Yang M, He Z, et al. Insights into the Functions of LncRNAs in Drosophila. Int J Mol Sci. 2019;20(18):4646.

28. Zhuo C, Jiang R, Lin X, Shao M. LncRNA H19 inhibits autophagy by epigenetically silencing of DIRAS3 in diabetic cardiomyopathy. Oncotarget. 2017:8(1):1429-37.

29. Chen ZH, Wang WT, Huang W, Fang $K$, Sun YM, Liu SR, et al. The IncRNA HOTAIRM1 regulates the degradation of PML-RARA oncoprotein and myeloid cell differentiation by enhancing the autophagy pathway. Cell Death Differ. 2016:24(2):212-24.

30. Kang Y, Song J, Kim D, Ahh C, Park S, Chun CH, et al. PCGEM1 stimulates proliferation of osteoarthritic synoviocytes by acting as a sponge for miR770. J Orthop Res. 2016;34(3):412-8.

31. Wang K, Liu CY, Zhou LY, Wang JX, Wang M, Zhao B, et al. APF IncRNA regulates autophagy and myocardial infarction by targeting miR-188-3p. Nat Commun. 2015;6:6779.

32. Ma ZB, Zhang J, Xu XR, Qu Y, Dong H, Dang J, et al. LncRNA expression profile during autophagy and Malat1 function in macrophages. PLoS One. 2019;14(8): e0221104.

33. Liu Y, Liu H, Liu S, Wang S, Jiang RJ, Li S. Hormonal and nutritional regulation of insect fat body development and function. Arch Insect Biochem Physiol. 2009;71(1):16-30.

34. Li S, Yu X, Feng Q. Fat Body Biology in the Last Decade. Annu Rev Entomol. 2019;64:315-33.

35. Taguchi S, Iwami M, Kiya T. Identification and characterization of a novel nuclear noncoding RNA, Fben-1, which is preferentially expressed in the higher brain center of the female silkworm moth, Bombyx mori. Neurosci Lett. 2011:496(3):176-80.

36. Wu Y, Cheng T, Liu C, Liu D, Zhang Q, Long R, et al. Systematic Identification and Characterization of Long Non-Coding RNAs in the Silkworm, Bombyx mori. PLoS One. 2016;11(1):e0147147.

37. Zhou QZ, Zhang B, Yu QY, Zhang Z. BmncRNAdb: a comprehensive database of non-coding RNAs in the silkworm, Bombyx mori. BMC Bioinformatics. 2016;17(1):370.

38. Zhou QZ, Fang SM, Zhang Q, Yu QY, Zhang Z. Identification and comparison of long non-coding RNAs in the silk gland between domestic and wild silkworms. Insect Sci. 2018;25(4):604-16.

39. Xu X, Wang K, Zha X. An antisense IncRNA functions in alternative splicing of Bmdsx in the silkworm, Bombyx mori. Biochem Biophys Res Commun. 2019;516(3):639-44.

40. Wang $H, H u H$, Xiang Z, Lu C, Dai F, Tong X. Identification and characterization of a new long noncoding RNA iab-1 in the Hox cluster of silkworm, Bombyx mori identification of iab-1. J Cell Biochem. 2019;120(10): 17283-92.

41. Zhang $\mathrm{S}$, Yin $\mathrm{H}$, Shen $\mathrm{M}$, Huang $\mathrm{H}$, Hou $\mathrm{Q}$, Zhang $\mathrm{Z}$, et al. Analysis of IncRNA-mediated gene regulatory network of Bombyx mori in response to BmNPV infection. J Invertebr Pathol. 2020;170,107323.

42. Xie K, Tian L, Guo X, Li K, Li J, Deng X, et al. BmATG5 and BmATG6 mediate apoptosis following autophagy induced by 20-hydroxyecdysone or starvation. Autophagy. 2016;12(2):381-96.

43. Database resources of the National Center for Biotechnology Information. Nucleic Acids Res. 2018;46(D1):D8-D13.

44. Ashburner M, Ball CA, Blake JA, Botstein D, Butler H, Cherry JM, et al. Gene ontology: tool for the unification of biology. Nat Genet. 2000;25(1):25-9.

45. Kanehisa M, Goto S. KEGG: Kyoto Encyclopedia of Genes and Genomes. Nucleic Acids Res. 2000;28(1):27-30

46. Kaneko $Y$, Yasanga T, Suzuki M, Sakurai S. Larval fat body cells die during the early pupal stage in the frame of metamorphosis remodelation in Bombyx mori. J Insect Physiol. 2011;57(12):1715-22.

47. Zheng $H$, Yang $X, X i$ Y. Fat body remodeling and homeostasis control in Drosophila. Life Sci. 2016;167:22-31.

48. Guo SY, Wu WM, Li SY, Liu Y, Ruan ZF, Ye MQ, et al. 20-Hydroxyecdysoneupregulated proteases involved in Bombyx larval fat body destruction. Insect Mol Biol. 2018:27(6):724-38.

49. Derrien T, Johnson R, Bussotti G, Tanzer A, Djebali S, Tilgner $H$, et al. The GENCODE v7 catalog of human long noncoding RNAs: analysis of their gene structure, evolution, and expression. Genome Res. 2012;22(9):1775-89.

50. White KP, Rifkin SA, Hurban P, Hogness DS. Microarray analysis of Drosophila development during metamorphosis. Science. 1999;286(5447):2179-84.

51. Beckstead RB, Lam G, Thummel CS. The genomic response to 20hydroxyecdysone at the onset of Drosophila metamorphosis. Genome Biol. 2005;6(12):R99.

52. Tian L, Guo E, Wang S, Liu S, Jiang RJ, Cao Y, et al. Developmental regulation of glycolysis by 20-hydroxyecdysone and juvenile hormone in fat body tissues of the silkworm, Bombyx mori. J Mol Cell Biol. 2010;2(5): 255-63.

53. Cohen-Kaplan V, Livneh I, Avni N, Cohen-Rosenzweig C, Ciechanover A. The ubiquitin-proteasome system and autophagy: Coordinated and independent activities. Int J Biochem Cell Biol. 2016;79:403-18.

54. Maruyama T, Noda NN. Autophagy-regulating protease Atg4: structure, function, regulation and inhibition. J Antibiot (Tokyo). 2017;71(1):72-8.

55. Betin VM, Lane JD. Caspase cleavage of Atg4D stimulates GABARAP-L1 processing and triggers mitochondrial targeting and apoptosis. J Cell Sci. 2009:122(Pt 14):2554-66

56. Cabrera S, Maciel M, Herrera I, Nava T, Vergara F, Gaxiola M, et al. Essential role for the ATG4B protease and autophagy in bleomycin-induced pulmonary fibrosis. Autophagy. 2015;11(4):670-84.

57. Tian L, Guo E, Diao Y, Zhou S, Peng Q, Cao Y, et al. Genome-wide regulation of innate immunity by juvenile hormone and 20hydroxyecdysone in the Bombyx fat body. BMC Genomics. 2010;11:549.

58. Klionsky DJ, Cuervo AM, Seglen PO. Methods for monitoring autophagy from yeast to human. Autophagy. 2007;3(3):181-206.

59. DeVorkin L, Gorski SM. LysoTracker staining to aid in monitoring autophagy in Drosophila. Cold Spring Harb Protoc. 2014;2014(9):951-8.

60. Wu X, Zhou $X$, Xiong L, Pei J, Yao X, Liang C, et al. Transcriptome Analysis Reveals the Potential Role of Long Non-coding RNAs in Mammary Gland of Yak During Lactation and Dry Period. Front Cell Dev Biol. 2020;8:579708. 
61. Kim D, Pertea G, Trapnell C, Pimentel H, Kelley R, Salzberg SL. TopHat2 accurate alignment of transcriptomes in the presence of insertions, deletions and gene fusions. Genome Biol. 2013;14(4):R36.

62. Guttman M, Garber M, Levin JZ, Donaghey J, Robinson J, Adiconis X, et al. Ab initio reconstruction of cell type-specific transcriptomes in mouse reveals the conserved multi-exonic structure of lincRNAs. Nat Biotechnol. 2010;28(5):503-10.

63. Trapnell C, Williams BA, Pertea G, Mortazavi A, Kwan G, van Baren MJ, et al. Transcript assembly and quantification by RNA-Seq reveals unannotated transcripts and isoform switching during cell differentiation. Nat Biotechnol. 2010;28(5):511-15.

64. Kong L, Zhang Y, Ye ZQ, Liu XQ, Zhao SQ, Wei L, et al. CPC: assess the protein-coding potential of transcripts using sequence features and support vector machine. Nucleic Acids Res. 2007;35(Web Server issue):W345-W349.

65. Punta M, Coggill PC, Eberhardt RY, Mistry J, Tate J, Boursnell C, et al. The Pfam protein families database. Nucleic Acids Res. 2012;40(Database issue): D290- D301.

66. Trapnell C, Pachter L, Salzberg SL. TopHat: discovering splice junctions with RNA-SEq. Bioinformatics. 2009;25(9):1105-11.

67. Kopp F, Mendell JT. Functional Classification and Experimental Dissection of Long Noncoding RNAs. Cell. 2018;172(3):393-407.

68. Zhan S, Dong Y, Zhao W, Guo J, Zhong T, Wang L, et al. Genome-wide identification and characterization of long non-coding RNAs in developmental skeletal muscle of fetal goat. BMC Genomics. 2016;17(1):666.

69. Young MD, Wakefield MJ, Smyth GK, Oshlack A. Gene ontology analysis for RNA-seq: accounting for selection bias. Genome Biol. 2010;11(2):R14.

70. Mao X, Cai T, Olyarchuk JG, Wei L. Automated genome annotation and pathway identification using the KEGG Orthology (KO) as a controlled vocabulary. Bioinformatics. 2005;21(19):3787-93.

71. Casati B, Terova G, Cattaneo AG, Rimoldi S, Franzetti E, de Eguileor M, et al. Molecular cloning, characterization and expression analysis of ATG1 in the silkworm, Bombyx mori. Gene. 2012;511(1):326-37.

72. Li S, Xu J, Xu X, Ma W, Tian L, Li K. Functional identification of Bombyx mori Atg13 in autophagy. Arch Insect Biochem Physiol. 2020;105(1):e21718.

73. Livak KJ, Schmittgen TD. Analysis of relative gene expression data using real-time quantitative PCR and the 2(-Delta Delta C(T)) Method. Methods. 2001;25(4):402-8.

74. Corpet F. Multiple sequence alignment with hierarchical clustering. Nucleic acids research. 1988;16(22):10881-90.

75. Robert $X$, Gouet P. Deciphering key features in protein structures with the new ENDscript server. Nucl Acids Res. 2014;42(W1):W320-W324.

\section{Publisher's Note}

Springer Nature remains neutral with regard to jurisdictional claims in published maps and institutional affiliations.

Ready to submit your research? Choose BMC and benefit from:

- fast, convenient online submission

- thorough peer review by experienced researchers in your field

- rapid publication on acceptance

- support for research data, including large and complex data types

- gold Open Access which fosters wider collaboration and increased citations

- maximum visibility for your research: over $100 \mathrm{M}$ website views per year

At BMC, research is always in progress.

Learn more biomedcentral.com/submissions 